\title{
The Hyers theorem via the Markov-Kakutani fixed point theorem
}

\author{
Barbara Przebieracz
}

To Professor Bogdan Bojarski

\begin{abstract}
We present an application of the Markov-Kakutani common fixed point theorem to the theory of stability of functional equation by proving some version of the Hyers theorem concerning approximate homomorphisms.
\end{abstract}

Mathematics Subject Classification. 39B82, 47H10, 46N99.

Keywords. Hyers theorem, Cauchy equation, stability, Markov-Kakutani fixed point theorem, approximate homomorphisms.

\section{Introduction}

One of the most celebrated results of the theory of common fixed points is a theorem proved independently by Markov [8] and Kakutani [7] (see also [9] and $[10])$.

Theorem 1.1 (Markov-Kakutani fixed point theorem). Let $Y$ be a linear topological space and let $K \subset Y$ be a nonempty convex compact subset of $Y$. Let $\mathcal{F}$ be a family of affine continuous self-mappings of $K$ such that $F \circ G=$ $G \circ F$ for $F, G \in \mathcal{F}$. Then there is a common fixed point $y \in K$ of family $\mathcal{F}$, i.e., $F(y)=y$, for every $F \in \mathcal{F}$.

The theorem of Hyers [5] was a partial answer to the problem posed by Ulam (see [12] and [13]): does there exist for an approximate homomorphism $\varphi$ a homomorphism which approximates $\varphi$ ? The result of Hyers initiated the works of many authors on the stability of functional equations (see [6]); it suffices to mention that his paper was cited several hundred times. The method used in [5], called the direct method or the Hyers sequences method, was applied in many papers; see the comments in [3]. The second basic approach to the stability of Cauchy functional equation involves the technic of invariant means and was introduced by Székelyhidi [11]. Let us mention also the paper [1] in which the authors noticed the relationship 
between Hyers sequences and the sequence of iterates of Lipschitz operator, therefore they proved the Hyers theorem via some generalization of the Banach contraction principle. Here we use the Markov-Kakutani fixed point theorem to prove the following.

Theorem 1.2 (Hyers' theorem). Let $(S,+)$ be an abelian semigroup, $\varepsilon \geq 0$ and $\varphi: S \rightarrow \mathbb{K}$, where $\mathbb{K} \in\{\mathbb{R}, \mathbb{C}\}$. Assume that

$$
|\varphi(x+y)-\varphi(x)-\varphi(y)| \leq \varepsilon, \quad x, y \in S .
$$

Then there exists an additive function $a: S \rightarrow \mathbb{K}$ such that

$$
|a(s)-\varphi(s)| \leq \varepsilon, \quad s \in S .
$$

The original Hyers theorem involves approximate homomorphisms between two normed spaces (codomain complete). However, taking into account the result of Gajda [4], it is sufficient to restrict ourselves to real or complex functions.

\section{Preliminaries}

In this section we recall some results from functional analysis (see [2]) and prove two lemmas.

For an arbitrary set $E$ let $X=\ell_{1}(E)$ be the space of all summable functions defined on $E$ with values in $\mathbb{K}$ (that is, the functions $f: E \rightarrow \mathbb{K}$ such that the set $E_{0}:=\{s \in E: f(s) \neq 0\}$ is at most countable and the series $\sum_{s \in E}|f(s)|:=\sum_{s \in E_{0}}|f(s)|$ is convergent), endowed with the norm

$$
\|f\|_{1}=\sum_{s \in E}|f(s)| .
$$

By $\ell_{\infty}(E)$ we denote the space of all bounded functions defined on $E$ with values in $\mathbb{K}$ endowed with supremum norm; that is,

$$
\|f\|_{\infty}=\sup _{s \in E}|f(s)| .
$$

For every $h \in \ell_{\infty}(E)$ and $f \in \ell_{1}(E)$ we have $f h \in \ell_{1}(E)$. Moreover, $\lambda_{h}: \ell_{1}(E) \rightarrow \mathbb{K}$, defined by $\lambda_{h}(f)=\sum_{s \in E} f(s) h(s)$, is a continuous linear functional on $\ell_{1}(E)$, i.e., $\lambda_{h} \in \ell_{1}(E)^{*}$. We have also $\|h\|_{\infty}=\left\|\lambda_{h}\right\|_{*}:=$ $\sup _{\|f\|_{1}=1}\left|\lambda_{h}(f)\right|$. Conversely, for every $\lambda \in \ell_{1}(E)^{*}$ there is exactly one $h \in \ell_{\infty}(E)$ such that $\lambda=\lambda_{h}$. The mapping

$$
\ell_{\infty}(E) \ni h \mapsto \lambda_{h} \in \ell_{1}(E)^{*}
$$

is an isometric isomorphism, therefore we can identify the space $\ell_{\infty}(E)$ with the space $\ell_{1}(E)^{*}$ and consider the space $\ell_{\infty}(E)$ with the weak ${ }^{*}$ topology; that is, the weakest topology in which the mappings $\ell_{1}(E)^{*} \ni \lambda \mapsto \lambda(f) \in \mathbb{K}$, $f \in \ell_{1}(E)$, are continuous. To shorten the notation we will write $Y=\ell_{\infty}(E)$ and $X=\ell_{1}(E)$. 
Lemma 2.1. Let $x \in E$, where $(E,+)$ is an abelian semigroup. The map $G: Y \rightarrow Y\left(Y=X^{*}\right.$ is considered with the weak* topology) given by $G(f)=$ $f(x+\cdot)$ is continuous.

Proof. Let $\left(f_{\alpha}\right)$ be a net in $Y$ convergent to $f \in Y$. Fix an arbitrary $u \in X$. In the set $E$ we consider an equivalence relation $\sim$ given by $s \sim t$ if and only if $x+s=x+t$. Let $[S]_{\sim}=\left\{S_{i}, i \in I\right\}$ be the set of all equivalence classes. Let us choose an $s_{i} \in S_{i}, i \in I$. Define $v(t):=\sum_{s \in S_{i}} u(s)$ if $t=x+s_{i}$, for some $i \in I$, and $v(t):=0$ if $t \neq x+s_{i}$ for every $i \in I$. It is easy to observe that $v \in X$. We assume that the net $\left(f_{\alpha}\right)$ is convergent to $f$, which gives $\lambda_{f_{\alpha}}(v) \rightarrow \lambda_{f}(v)$. After some elementary calculations (using the form of functionals in $\left.X^{*}\right)$, we get

$$
\sum_{s \in S} G\left(f_{\alpha}\right)(s) u(s) \rightarrow \sum_{s \in S} G(f)(s) u(s) .
$$

Since $u \in X$ is arbitrary, we proved that $\lambda_{G\left(f_{\alpha}\right)} \rightarrow \lambda_{G(f)}$ in the weak* topology. Hence $\left(G\left(f_{\alpha}\right)\right)$ tends to $G(f)$, which ends the proof.

Lemma 2.2. Let $x \in E$. The map $G: Y \rightarrow Y$ given by $G(f)=f(x)$ is continuous (with respect to the weak* topology in $Y$ ).

Proof. Assume that a net $\left(f_{\alpha}\right)$ is convergent to $f$ with respect to the weak* topology. In fact, this means that the net $\left(\lambda_{f_{\alpha}}\right)$ converges weakly* to $\lambda_{f}$ which easily yields that $\left(f_{\alpha}\right)$ is pointwise convergent to $f$, in particular, the net $\left(f_{\alpha}(x)\right)$ converges to $f(x)$ in $\mathbb{K}$. Hence we may infer that the net $\left(G\left(f_{\alpha}\right)\right)$ is weakly* convergent to $G(f)$ in $Y$.

\section{Proof of Theorem 1.2}

Let $(S,+)$ be an abelian semigroup, $\varepsilon \geq 0$ and $\varphi: S \rightarrow \mathbb{K}$, where $\mathbb{K} \in\{\mathbb{R}, \mathbb{C}\}$. Assume that

$$
|\varphi(x+y)-\varphi(x)-\varphi(y)| \leq \varepsilon, \quad x, y \in S .
$$

Denote by $Y$ the space $\ell_{\infty}(S)$. Since $Y=X^{*}$, where $X=\ell_{1}(S)$, we can consider $Y$ with the weak* topology. With this topology $Y$ is a linear topological space.

For every $x \in S$ we define a map $T_{x}: Y \rightarrow Y$ by the formula

$$
T_{x}(f):=f(x+\cdot)+\varphi(x+\cdot)-f(x)-\varphi(x)-\varphi(\cdot) .
$$

(We see that $T_{x}(f) \in Y$, since $f \in Y$ and (3.1) holds.) Moreover, $T_{x}$ are affine, that is,

$$
T_{x}(t f+(1-t) g)=t T_{x}(f)+(1-t) T_{x}(g), \quad f, g \in Y, t \in[0,1] .
$$


Notice also that for $x, y, z \in S$ and $f \in Y$ we have

$$
\begin{aligned}
& T_{x}\left(T_{y}(f)\right)(z) \\
&= T_{y}(f)(x+z)+\varphi(x+z)-T_{y}(f)(x)-\varphi(x)-\varphi(z) \\
&=(f(y+x+z)+\varphi(y+x+z)-f(y)-\varphi(y)-\varphi(x+z))+\varphi(x+z) \\
&-(f(y+x)+\varphi(y+x)-f(y)-\varphi(y)-\varphi(x))-\varphi(x)-\varphi(z) \\
&= f(y+x+z)+\varphi(y+x+z)-f(y+x)-\varphi(y+x)-\varphi(z),
\end{aligned}
$$

which means that

$$
T_{x} \circ T_{y}=T_{y+x}, \quad x, y \in S .
$$

Moreover, for every $x \in S$ the map $T_{x}: Y \rightarrow Y$ is continuous with respect to the weak* topology, since it is the sum of the mappings $Y \ni f \mapsto$ $f(x) \in Y, Y \ni f \mapsto f(x+\cdot) \in Y$, which are continuous (see Lemmas 2.1 and $2.2)$, and $Y \ni f \mapsto(\varphi(x+\cdot)-\varphi(x)-\varphi(\cdot)) \in Y$ which is constant.

Now let us define the set

$$
\mathcal{C}:=\left\{f \in Y:\|f\|_{\infty} \leq \varepsilon,\left\|T_{x}(f)\right\|_{\infty} \leq \varepsilon, x \in S\right\} .
$$

Observe that $\mathbf{0} \in \mathcal{C}$, hence $\mathcal{C}$ is nonempty (cf. (3.1)). Moreover, $\mathcal{C}$ is convex. Indeed, assume that $f, g \in \mathcal{C}$ and $t \in[0,1]$. Then

$$
\|t f+(1-t) g\|_{\infty} \leq t\|f\|_{\infty}+(1-t)\|g\|_{\infty} \leq t \varepsilon+(1-t) \varepsilon=\varepsilon .
$$

Similarly, since $T_{x}$ are affine, we get

$$
\begin{aligned}
\left\|T_{x}(t f+(1-t) g)\right\|_{\infty} & =\left\|t T_{x}(f)+(1-t) T_{x}(g)\right\|_{\infty} \\
& \leq t\left\|T_{x}(f)\right\|_{\infty}+(1-t)\left\|T_{x}(g)\right\|_{\infty} \\
& \leq t \varepsilon+(1-t) \varepsilon=\varepsilon
\end{aligned}
$$

Observe also that $\mathcal{C}$ is invariant under every $T_{x}$ : fix $x \in S$ and $f \in \mathcal{C}$, then $\left\|T_{x}(f)\right\|_{\infty} \leq \varepsilon$ and $\left\|T_{y}\left(T_{x}(f)\right)\right\|_{\infty}=\left\|T_{x+y} f\right\|_{\infty} \leq \varepsilon, y \in S$ (cf. (3.2)), hence $T_{x}(\mathcal{C}) \subset \mathcal{C}$.

Let $K$ be the weak* closure of the set $\mathcal{C}$. Then $K$ is nonempty, convex (since $\mathcal{C}$ is nonempty and convex), $T_{x}(K) \subset K$, for every $x \in S$ (since $\mathcal{C}$ is invariant and $T_{x}$ are continuous); moreover, $K$ is compact, as a closed subset of $B_{\varepsilon}:=\left\{f \in Y:\|f\|_{\infty} \leq \varepsilon\right\}$, which is weak*-compact according to the Banach-Alaoglu theorem. We have shown that all the assumptions of Markov-Kakutani fixed point theorem are satisfied (with $\mathcal{F}:=\left\{T_{x}: x \in S\right\}$ ). Thereby there exists an $f \in K$ such that $T_{x}(f)=f$ for every $x \in S$. This means that

$$
f(x+y)+\varphi(x+y)-f(x)-\varphi(x)-\varphi(y)=f(y), \quad x, y \in S .
$$

Put $a:=f+\varphi$. It is easily seen that $a: S \rightarrow \mathbb{K}$ is additive and $\|a-\varphi\|_{\infty}=$ $\|f\|_{\infty} \leq \varepsilon$, since $f \in K \subset B_{\varepsilon}$. The proof is finished. 


\section{Acknowledgements}

This work was supported by the Department of Mathematics, University of Silesia, Katowice, Poland (Iterative Functional Equations and Real Analysis Program).

The author gratefully acknowledges the many helpful suggestions of an anonymous referee.

\section{References}

[1] L. Cădariu and V. Radu, On the stability of the Cauchy functional equation: A fixed point approach. In: Iteration Theory (ECIT '02), Grazer Math. Ber. 346, Karl-Franzens-Univ. Graz, Graz, 2004, 43-52.

[2] M. Day, Normed Linear Spaces. 3rd ed., Ergebnisse der Mathematik und ihrer Grenzgebiete, Band 21, Springer-Verlag, New York, 1973.

[3] G. L. Forti, Comments on the core of the direct method for proving Hyers-Ulam stability of functional equations. J. Math. Anal. Appl. 295 (2004), 127-133.

[4] Z. Gajda, On the stability of the Cauchy equation on semigroups. Aequationes Math. 36 (1988), 76-79.

[5] D. H. Hyers, On the stability of the linear functional equation. Proc. Natl. Acad. Sci. USA 27 (1941), 222-224.

[6] D. H. Hyers, G. Isac and Th. M. Rassias, Stability of Functional Equations in Several Variables. Birkhäuser Boston, Boston, MA, 1998.

[7] S. Kakutani, Two fixed-point theorems concerning bicompact convex sets. Proc. Imp. Acad. 14 (1938), 27-31.

[8] A. Markov, Quelques théorèmes sur ensembles abéliens. C. R. (Doklady) Acad. Sci. URSS N.S. 1 (1936), 311-313.

[9] I. Namioka, Kakutani-type fixed point theorems: A survey. J. Fixed Point Theory Appl. 9 (2011), 1-23.

[10] W. Rudin, Functional Analysis. 2nd ed., International Series in Pure and Applied Mathematics, McGraw-Hill, New York, 1991.

[11] L. Székelyhidi, Remark 17. In: The Twenty-Second International Symposium on Functional Equations, December 16-December 22, 1984, Oberwolfach, Germany, Aequationes Math. 29 (1985), 95-96.

[12] S. M. Ulam, Problems in Modern Mathematics. Science Editions, John Wiley \& Sons, New York, 1964.

[13] S. M. Ulam, A Collection of Mathematical Problems. Interscience Tracts in Pure and Applied Mathematics 8, Interscience Publishers, New York, 1960.

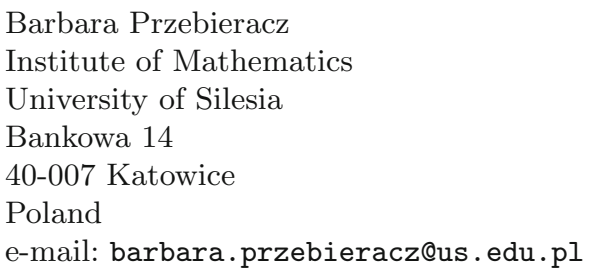

Open Access This article is distributed under the terms of the Creative Commons Attribution License which permits any use, distribution, and reproduction in any medium, provided the original author(s) and the source are credited. 